\title{
O concreto no processo de industrialização da arquitetura: da pré-fabricação à fabricação digital
}

DANIEL DE SOUZA GONÇALVES - Arquiteto e Mestrando em Design
PAUl0 EDUARDO FONSECA DE CAMPOS - Professor Associado
Faculdade de Arquitetura e Urbanismo da Universidade de São Paulo (FAU USP)

\section{RESUMO}

O PROCESSO DE INDUSTRIALIZAÇÃO DA CONSTRUÇÃO CIVIL NO BRASIL, POR MEIO DA INCORPORAÇÃO DE NOVAS TECNOLOGIAS, É UM TEMA AMPLAMENTE DISCUTIDO NA ATUALIDADE, DENTRO E FORA DA ACADEMIA. MARCANDO MOMENTOS DE GRANDE AVANÇO TECNOLÓGICO NO CAMPO DA PRÉ-FABRICAÇÃO, PODE-SE DESTACAR TRÊS EXPERIÊNCIAS RELEVANTES NA CONSTRUÇÃO CIVIL BRASILEIRA EM QUE SE FEZ USO DA TECNOLOGIA DE MATERIAIS, ESPECIALMENTE DO CONCRETO, ALIADAS A PROJETOS PARTICIPATIVOS PARA O DESENVOLVIMENTO DE SOLUÇÕES VOLTADAS A COMUNIDADES CARENTES. DO PIONEIRISMO DAS

EXPERIÊNCIAS dE JoÃo FilgueIRAS Lima (LelÉ), PASSANdo PELOS PROJEtos SOCIAIS do Programa Ibero-Americano de CoOperação CYTED (Ciencia y Tecnología para el Desarrollo) e chegando ao panorama ATUAL, JÁ FAZENDO USO DE MEIOS DIGITAIS DE FABRICAÇÃO, ESTE ARTIGO VISA ESTUDAR COMO O PROCESSO DE INDUSTRIALIZAÇÃO DA CONSTRUÇÃO CIVIL NO BRASIL AVANÇOU NAS ÚLTIMAS DÉCADAS E COMO AS NOVAS TECNOLOGIAS COMPUTACIONAIS E DE MATERIAIS, ESPECIALMENTE DO CONCRETO, PODEM INDICAR FUTUROS CAMINHOS PARA O DESENVOLVIMENTO.

Palavras-chave: industrialização, concreto, fabricação digital, pré-fabricação.

\section{INTRODUÇÃO}

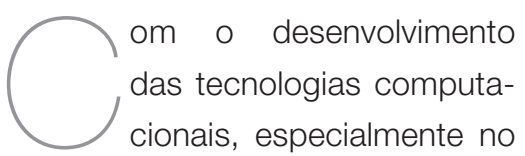
final do século XX e início do XXI, novos meios de produção, antes inimagináveis, se tornaram realidade dentro dos parques industriais. Com o uso do controle computacional, novas máquinas possibilitaram um grande aumento de produtividade e qualidade nos produtos manufaturados, além da redução de preços para o consumidor final, como consequência do aumento da escala (GERSHENFELD, 2012). Nesse contexto de modernização dos meios de produção, diferentemente da indústria tradicional, o setor da construção civil ainda apresenta avanços tímidos no desenvolvimento e incorporação de novas tecnologias. Especialmente no caso de países em desenvolvimento, como o Brasil, a construção civil ainda se baseia em processos de produção majoritariamente tradicionais, resultando não apenas em baixa produtividade, com a exploração de mão de obra barata e pouco qualificada, como também em edificações cuja qualidade apresenta sérias deficiências, comumente apresentando problemas construtivos ao longo de sua vida útil.

Apesar do cenário de defasagem tecnológica da construção civil brasileira, avanços significativos puderam ser observados ao longo das últimas décadas, tendo como foco principal o desenvolvimento de novos materiais e processos para sistemas construtivos pré-fabricados, majoritariamente empregando o concreto como insumo principal. Visando compreender como se deram as experiências de industrialização da construção no País, este artigo destaca três períodos com produções arquitetônicas socialmente relevantes, marcados pelo uso de novas tecnologias nos processos construtivos.

$\mathrm{O}$ primeiro período remete ao que pode ser considerado um dos exemplos mais profícuos da industrialização na arquitetura, com os sistemas construtivos leves em argamassa armada de João Filgueiras Lima (Lelé). 
Na sequência, são abordadas as experiências em pré-fabricação com o material sucedâneo da argamassa armada, o microconcreto de alto desempenho (MicroCAD), realizadas no âmbito do Programa Ibero-Americano de Cooperação "Ciência e Tecnologia para o Desenvolvimento" (CYTED Ciencia y Tecnología para El Desarrollo), fazendo uso de soluções construtivas voltadas a comunidades carentes. Por fim, o terceiro período a ser abordado traz as experiências atuais já fazendo uso da fabricação digital para a produção arquitetônica de componentes e sistemas desenvolvidos com o auxílio de softwares para modelagem paramétrica.

Por meio da revisão desses três momentos importantes na industrialização da construção civil, este artigo aborda como essas experiências exploraram possíveis caminhos para o desenvolvimento do setor, refletindo a respeito de como as novas tecnologias computacionais aliadas à tecnologia de materiais podem oferecer uma perspectiva de futuro à produção arquitetônica nacional.

\section{DA PRÉ-FABRICAÇÃO À FABRICAÇÃO DIGITAL}

\section{I 0 expoente da pré-fabricação no Brasil}

Ao ser convidado em 2010 para ir a Portugal proferir uma palestra no $1^{\circ}$ Congresso Internacional da Habitação no Espaço Lusófono (ClHEL), o arquiteto carioca João Filgueiras Lima (Lelé) já carregava em sua trajetória experiências singulares em processos de pré-fabricação na arquitetura, aos quais se dedicava desde o início de sua atividade como arquiteto na década de 1950. Em uma entrevista, transcrita na íntegra pelo website de arquitetura Vitruvius ${ }^{1}$, ao ser questionado sobre sua visão alternativa com relação aos sistemas tradicionais de construção, Lelé deixa clara a sua intenção em produzir peças pré-fabricadas com o uso de tecnologias acessíveis, para que populações com demandas específicas pudessem, assistidas por um profissional arquiteto, ser protagonistas no processo de produção:

"O qUE DEFENDEMOS É JUSTAMENTE A DIVULGAÇÃO DE UM SISTEMA CONSTRUTIVO QUE PERMITISSE QUASE A AUTOCONSTRUÇÃO. É COMO QUE UM LEGO QUE NÓS MONTÁSSEMOS A PARTIR DESSES COMPONENTES, DE UMA FORMA SIMPLES, QUE PUDESSE SER APRENDIDA POR UMA MÃO DE OBRA COM POUCA QUALIFICAÇÃO, E DE UMA FORMA RÁPIDA." (LELÉ, 2010)
Esse pensamento foi posto em prática por Lelé em 1979 com sua participação na Companhia de Renovação Urbana de Salvador (RENURB), onde desenvolveu projetos de infraestrutura urbana para as comunidades da capital [baiana] (GUIMARÃES, 2010). O projeto de drenagem (Figura 1) utilizou como material a argamassa armada para criar peças pré-fabricadas leves de pequena espessura e alta resistência mecânica. Lelé dá uma boa definição da técnica quando questionado sobre seu uso extensivo ao longo dos anos de sua produção:

"A ARGAMASSA ARMADA NÃO É NENHUMA NOVIDADE, FOI EXPLORADA, E MUITO BEM MANUSEADA, PELO ENGENHEIRO FRANCÊS JOSEPH-LOUIS LAMBOT HÁ DUZENTOS ANOS ATRÁS. EXISTEM ATÉ EXPERIÊNCIAS FEITAS COM EMBARCAÇÕES NESSA ÉPOCA. No Museu Francês da ConstruÇÃO EXISTE UM EQUIPAMENTO DESSES QUE FOI RESGATADO DO FUNDO

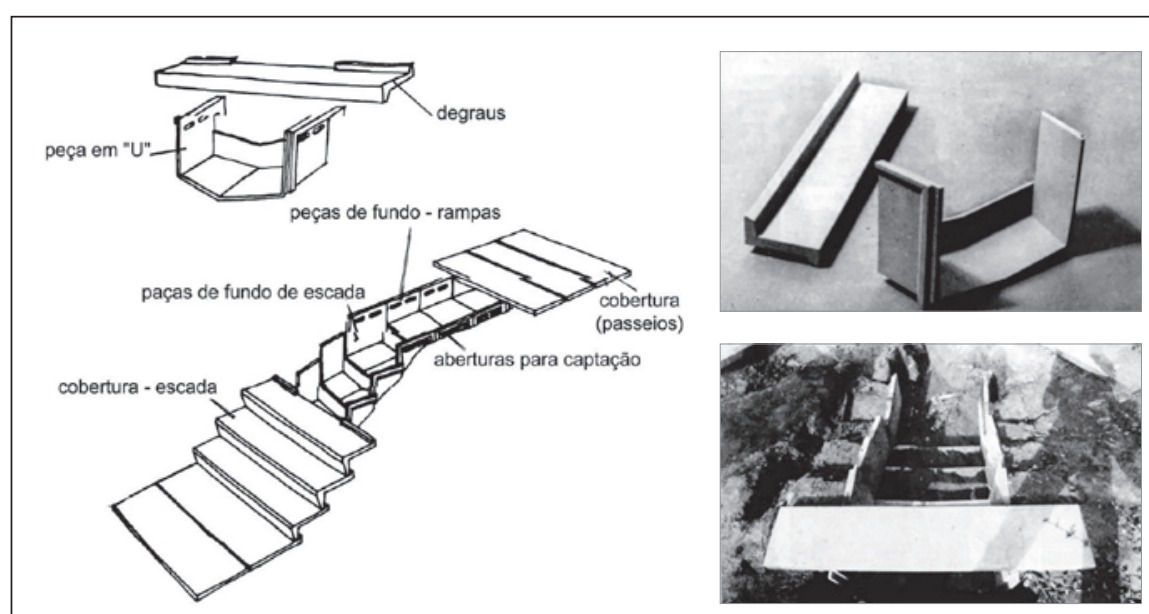

Figura 1

Projeto de drenagem de Lelé na RENURB, 1980

Fonte: Guimarães (2010) 
DE UM LAGO, E QUE EXPRESSA PERFEITAMENTE TODA A QUALIDADE TÉCNICA DO MATERIAL. JÁ NA DÉCADA DE 1940, Pier LUigi NerVI utilizou BASTANTE A ARGAMASSA ARMADA, QUE ELE CHAMAVA DE "FERROCIMENTO”. EXISTEM ALGUMAS NUANCES ENTRE ESSE PRODUTO, QUE FOI EXPLORADO POR LAMBOT, DEPOIS POR NERVI, E O QUE PRATICAMOS AGORA. NA VERDADE, NESSAS DUAS EXPERIENNCIAS, A ARGAMASSA ARMADA TINHA UM TEOR DE AÇO MUITO MAIOR DO QUE A QUE NÓS USAMOS HOJE, ATÉ POR UMA QUESTÃO DE CUSTO. As PROPRIEDADES MECÂNICAS DA ARGAMASSA ARMADA DIFEREM UM POUCO DAS DO CONCRETO ARMADO, PORQUE EXISTE UMA PRESENÇA MAIOR DAS ARMADURAS - QUE CHAMAMOS UMA LIGAÇÃO DE "PELE" PORTANTO TEM DE HAVER UM CONTATO MAIOR NAS ARMADURAS DE aÇO ENTRE O CIMENTO E A TELA. AO PASSO QUE NO CONCRETO ARMADO, OS DOIS MATERIAIS TRABALHAM DE FORMA COMPLETAMENTE DIFERENTE, SÃO INDEPENDENTES: O AÇO TRABALHANDO A TRAÇÃO, E A ARGAMASSA TRABALHANDO A COMPRESSÃO." (LELÉ, 2010)

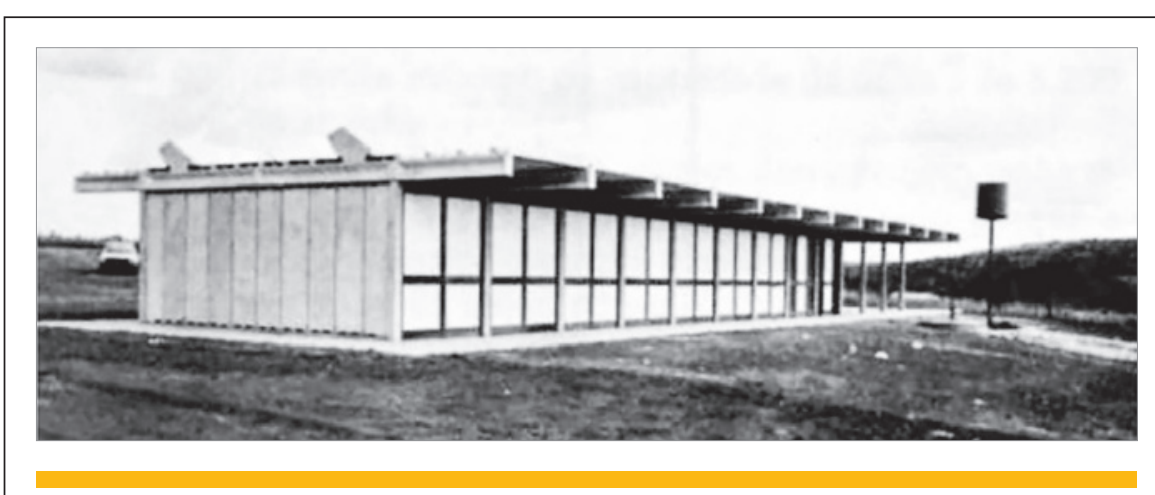

Figura 2

Escolas Transitórias de Abadiânia, projeto de Lelé

Fonte: Lima, João Filgueiras Escola Transitória - Brasília, MEC/CEDATE, 1984

A argamassa armada, como definida por Lelé, representou um grande avanço tecnológico na produção de elementos pré-moldados, mesmo ainda se tratando de uma técnica parcialmente artesanal. As experiências de Lelé com o material resultaram no aumento da qualidade de execução das obras por meio da participação popular, com o acompanhamento do projeto à execução. As Escolas Transitórias em Abadiânia/GO (Figura 2), desenvolvidas em 1982, são exemplos de projeto em que a participação popular e o treinamento de mão de obra local foram fundamentais para a qualidade do projeto. Foram utilizadas formas metálicas para a produção de peças de argamassa armada no próprio canteiro de obras, primorosamente dispensando o uso de qualquer maquinário específico para o deslocamento e montagem. (TRIGO, 2009) O resultado dessa experiência foi a construção de escolas locais pelas próprias mãos da população, que agora treinada e consciente do processo, pôde replicá-lo em novas ações para demandas locais.

Além dessas experiências, Lelé ainda desenvolveu projetos com sis- 
conceituados, além de empresários, e o apoio de entidades de fomento à pesquisa, como o CNPq-Conselho Nacional de Desenvolvimento Científico e Tecnológico (FONSECA DE CAMPOS, 2013).

Ao longo dos cinco anos de atuação, o projeto se dedicou à pesquisa e desenvolvimento de projetos utilizando o microconcreto de alto desempenho para a produção de peças pré-fabricadas leves, projetadas para demandas locais em diversas comunidades da América Latina. Tecnologia considerada como sucessora da argamassa armada, o MicroCAD é fruto do avanço tecnológico na qualidade do concreto, caracterizando-se como um material de baixa relação entre água e aglomerante (cimento + aditivos) e a eventual adição de materiais cimentícios suplementares (sílica ativa, metacaulim etc.). Apresentando entre as suas propriedades alta resistência, baixíssima permeabilidade e trabalhabilidade adequada à produção de componentes pré-fabricados leves, devido ao uso de aditivos superplastificantes redutores de água, o MicroCAD armado representa um avanço nos resultados anteriormente obtidos com a argamassa armada tradicional, tornando possível o equacionamento de problemas vinculados, particularmente, à durabilidade. (Figura 3).

Dentre os vários projetos desenvolvidos pelo grupo, destaca-se as calçadas drenantes projetadas pelo professor Paulo Eduardo Fonseca de Campos, da FAUUSP, um dos autores do presente artigo, inspiradas nos projetos de drenagem desenvolvidos por Lelé no final da década de 1970 em Salvador/BA, e anteriormente

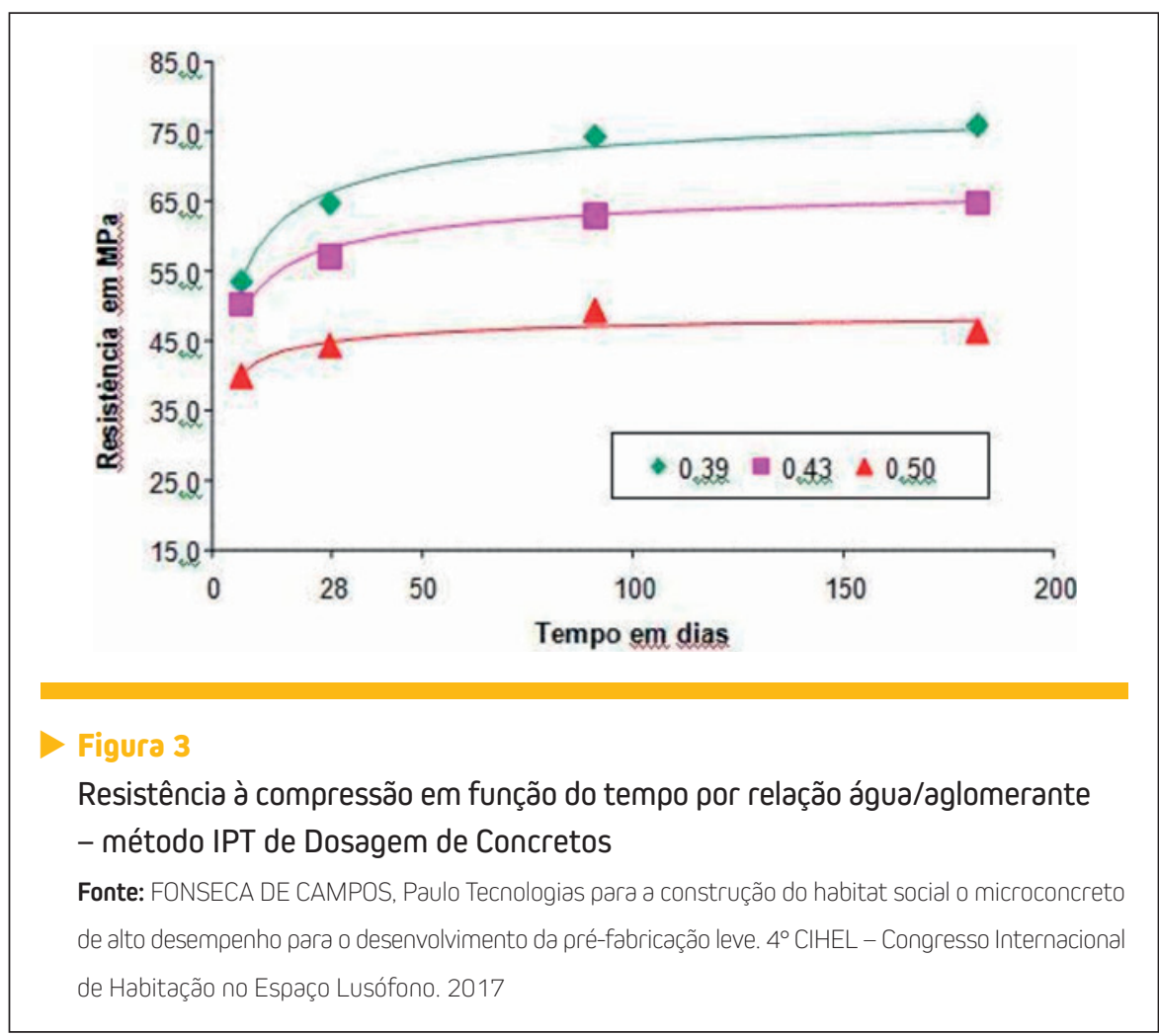

executados em argamassa armada. $\mathrm{Na}$ nova proposta, utilizam-se fôrmas simples de compensado naval que podem ser executadas por meio de carpintaria convencional, possibilitando o emprego de mão de obra local das comunidades. A moldagem e a desenforma das peças de MicroCAD seguem os mesmos processos empregados na produção de argamassa armada, sendo de fácil execução e gerando peças mais leves e resistentes para serem empregadas nas intervenções.

Como nos trabalhos desenvolvidos por Lelé, os projetos desenvolvidos no âmbito do Projeto MicroCAD/CYTED tiveram o arquiteto como agente de mudança na lógica tradicional de produção, incorporando novas tecnologias que possibilitaram a participação da população de forma ativa na construção de seu habitat social.

\subsection{A fabricação digital na construção civil: cenários $\in$ perspectivas}

As primeiras experiências com máquinas controladas numericamente por computador ocorreram na década de 1970, nos laboratórios do Massachusetts Institute of Technology (MIT), nos Estados Unidos da América. Inicialmente aplicado à indústria aeronáutica, o controle numérico computacional (CNC)

Tabela 1 - Dosagens para argamassa armada

Dosagens de referência

Consumo Areia Fator a/

de cimento (massa) aglomerante

$\begin{array}{ccc}\text { Traço rico } & 1,5 & 0,39 \\ \text { Traço médio } & 2,0 & 0,43 \\ \text { Traço pobre } & 2,5 & 0,50\end{array}$


rapidamente foi aplicado nas linhas de montagem da indústria tradicional, resultando no aumento na produtividade e, consequentemente, na substituição do trabalho humano pelas máquinas (GERSHENFELD, 2012). Com a popularização da informática na virada do milênio, produzir elementos customizados usando máquinas de corte a laser, fresadoras e impressoras 3D tornou-se algo acessível a diversas indústrias, mesmo no caso da construção civil.

O arquiteto americano Frank Gehry é reconhecido como o pioneiro no emprego da fabricação digital na arquitetura, sendo seu "Peixe" em Barcelona a primeira experiência com peças pré-fabricadas na arquitetura, produzidas com o uso do software paramétrico CATIA. Fabricada em chapa metálica perfurada, a escultura é uma amostra da plasticidade e complexidade das formas

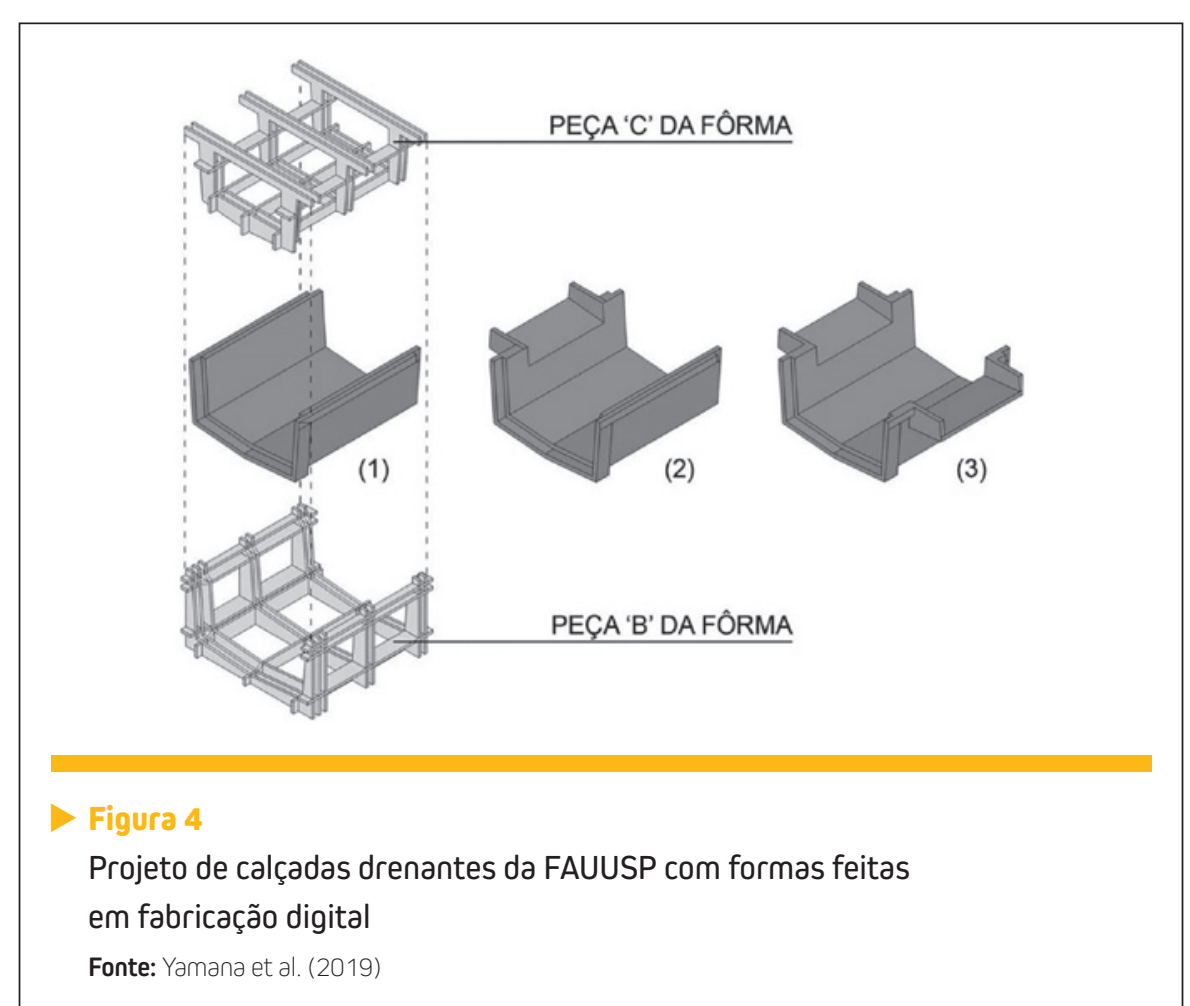

possibilitadas pela fabricação digital, explorando novos modos de se projetar. O projeto foi desenvolvido segundo o conceito de file-to-factory, onde o tradicional conjunto de plantas e cortes é substituído pelo modelo tridimensional paramétrico, com todas as informações necessárias à produção por uma máquina CNC (FONSECA DE CAMPOS e LOPES, 2017). A produção de elementos com fabricação digital pode se dar por métodos aditivos, subtrativos ou conformativos, caracterizando-se respectivamente pela deposição de um material, desgaste ou alteração de sua forma. No caso do "Peixe" de Frank Gehry, executou-se um processo subtrativo, ao cortar chapas metálicas através de uma máquina CNC, e posteriormente, conformativo, para a obtenção das curvaturas desejadas.

São várias as possibilidades de se utilizar a fabricação digital na prodas "calçadas drenantes" (FIGURA 4) replicou a experiência prévia acumulada no Projeto MicroCAD/ CYTED, agora junto a uma comunidade carente da Zona Leste de São Paulo/Capital. O projeto não prevê a produção das peças finais com o uso da fabricação digital, mas sim a fabricação das fôrmas em compensado naval com o uso de cortadoras a laser, facilitando assim a construção dos moldes. A comunidade envolvida participou, inicialmente, do processo de produção e montagem das peças constituintes dos moldes, compreendendo como utilizar a fabricação digital para a resolução de problemas locais. (YAMANA et al., 2019) 


\section{CONCLUSÃO}

Ao se analisar os três períodos aqui apresentados, pode-se reconhecer que a industrialização da construção civil no Brasil experimentou avanços significativos voltados a soluções para problemas urbanos desafiadores na área do habitat.

Nas obras de Lelé, a pré-fabricação leve apresenta-se como uma solução adaptável às realidades locais por meio da produção participativa junto à população. Mesmo com suas limitações tecnológicas, a argamassa armada foi um marco no desenvolvimento do concreto armado ao propor uma nova forma de aplicação deste material tão conhecido e amplamente utilizado no País, resultando daí a possibilidade de utilização da pré-fabricação sob a forma de peças mais leves e de fácil manuseio e montagem.

Décadas depois, as experiências do Programa CYTED com o microconcreto de alto desempenho (MicroCAD) apresentam uma releitura da técnica da argamassa armada de Lelé, só possível devido aos avanços ocorridos na ciência dos materiais e com os adventos do concreto de alto desempenho e dos compósitos de base cimentícia. Desse modo, a incorporação de novas tecnologias aos processos de produção gerou uma possibilidade de ganho expressivo na produtividade e na qualidade da construção industrializada, além de tornar a pré-fabricação leve menos complexa, permitindo que populações carentes possam participar ativamente dos projetos de melhorias urbanas a serem implantados nos locais onde vivem.

Por fim, a fabricação digital, já largamente utilizada no setor industrial convencional, começa a ter suas primeiras experimentações no setor da indústria da construção civil, aspirando ganhos de produtividade através da modernização dos processos. Como apresentado, o Contour Crafting, por exemplo, fazendo uso de método aditivo baseado na impressão 3D em concreto, é uma das vertentes que vêm sendo pesquisadas em vários centros de pesquisa pelo mundo. Afora esta, há também iniciativas locais de desenvolvimento de fôrmas para a criação de peças pré-fabricadas, utilizando a fabricação digital como oportunidade para ampliar a participação popular particularmente nos processos de produção social do habitat.

Conclui-se, portanto, que o processo de industrialização da construção civil no Brasil, apesar de ainda estar longe de se consolidar, já tem em seu histórico um retrospecto de grande relevância, caso se considere as ações empreendidas por grupos de pesquisa e empresas que se ocupam da arquitetura industrializada como forma de avanço na produção do edifício e da infraestrutura urbana. Para tanto, foi indispensável o desenvolvimento da tecnologia de materiais, especialmente do concreto, para que se viabilizasse a criação de elementos pré-fabricados delgados e leves, adaptados às demandas e realidades locais, permitindo a participação da população no processo de projeto e produção. Nesse cenário, a fabricação digital se apresenta como uma ferramenta poderosa para a introdução de inovações tecnológicas no setor da habitação, principalmente em projetos sociais locais, possibilitando um caminho viável para o desenvolvimento de soluções mais eficientes e participativas.

\section{DREFER̂NCIAS BIBLIOGRÁFICAS}

[1] FONSECA DE CAMPOS, Paulo Eduardo. Microconcreto de alto desempeño: la tecnología del MicroCAD aplicada en la construcción del hábitat social. [S.l: s.n.], 2013.

[2] FONSECA DE CAMPOS, Paulo Eduardo; LOPES, Eduardo. A fabricação digital aplicada à construção industrializada/ estado da arte e perspectivas de desenvolvimento. 2017. Revista Concreto \& Construções, n.85, p. 22-29.

[3] GERSHENFELD, Neil. How to Make Almost Anything - The Digital Fabrication Revolution. Foreign Affairs 91, no. 6, 2012.

[4] GUIMARÃES, Ana Gabriella Lima. A obra de João Filgueiras Lima no contexto da cultura arquitetônica contemporânea. 2010. Tese (Doutorado em História e Fundamentos da Arquitetura e do Urbanismo) - Faculdade de Arquitetura e Urbanismo, Universidade de São Paulo, São Paulo, 2010.

[5] HWANG, Dooil; KHOSHNEVIS, Behrokh. Concrete wall fabrication by contour crafting. In: ISARC 200421 st International Symposium on Automation and Robotics in Construction, 2004.

[6] TRIGO, Cristina Câncio. Pré-fabricados em argamassa armada: material, técnica e desenho de componentes desenvolvidos por Lelé. 2009. Dissertação (Mestrado em Tecnologia da Arquitetura) - Faculdade de Arquitetura e Urbanismo, Universidade de São Paulo, São Paulo, 2009.

[7] YAMANA, D. N.; MEDEIROS, J.; LOPES, E. I.; FONSECA DE CAMPOS, P. E. Calçadas Drenantes: intervenções físicas com desenvolvimento social. Gestão \& Tecnologia de Projetos, [S. I.], v. 14, n. 1, p. 9-24, 2019. 